\title{
Treatment centre size, entry to trials, and survival in acute lymphoblastic leukaemia
}

\author{
C A STILLER AND G J DRAPER \\ Childhood Cancer Research Group, Department of Paediatrics, University of Oxford
}

SUMMARY In a population based series of 4070 children with acute lymphoblastic leukaemia treated in Britain during 1971-82, the five year survival rate improved from $37 \%$ for those treated in $1971-3$ to $66 \%$ in $1980-2$. During the same period there was an increase in the proportion of children included in the Medical Research Council trials and an increase in the proportion who were treated at centres with an average of at least six new patients per year. Survival rates were significantly higher for children who were included in the trials than for those who were not. Among children not in the trials, the survival rate was highest at centres treating at least six children per year and lowest at those with fewer than one per year. The increase in the numbers of children treated according to standardised protocols, particularly within controlled clinical trials, has had a major impact on the mortality from childhood acute lymphoblastic leukaemia.

In Britain during 1971-82, with a population of about 13000000 children below age 15 , about 350 cases per year of acute lymphoblastic leukaemia were notified to the National Registry of Childhood Tumours maintained by the Childhood Cancer Research Group. Large numbers of these children with acute lymphoblastic leukaemia were included in the United Kingdom Acute Lymphoblastic Leukaemia (UKALL) trials, in which various combinations of chemotherapy and radiotherapy have been evaluated. Details of the protocols and results of these trials have been presented in a series of publications from the Medical Research Council Working Party on Leukaemia in Childhood. ${ }^{1-8}$

During 1971-82 most major treatment centres entered patients into most of the UKALL trials, and there has been a continuing process of concentration of treatment at such centres. For part of the period some centres used the trial regimens or similar ones, or carried out pilot studies of further modifications, without entering patients into the current trials. Hospitals other than specialist centres also adopted the improved forms of treatment without necessarily entering patients into the trials. By the end of the period under review, virtually all children with acute lymphoblastic leukaemia were treated according to standardised protocols.

Dramatic improvements in survival rates were achieved in the earliest of these trials, but little further improvement in rates of remission or survival was achieved among patients entered into the trials during 1972-9. ${ }^{8}$ Trials conducted in the United States and Germany during the 1970s achieved higher success rates than the contemporaneous UKALL trials. As a result, the UKALL VIII protocol was introduced in 1980; this was based on a protocol developed by the United States Children's Cancer Study Group. The early results of this trial show considerable improvements on the preceding UKALL trials. ${ }^{8}$

In this paper we examine the trends in referral rates to specialist centres and in the proportions of children included in the trials, and the survival rates for children treated both inside and outside the trials and at centres of different sizes.

\section{Patients and methods}

The Childhood Cancer Research Group has received copies of all notifications for children aged under 15 who had leukaemia diagnosed during 1971-82 and were reported to the national cancer registration schemes in England, Scotland, and Wales. During the same period, copies of all notifications for children entered in the Medical Research Council UKALL trials were also received. Additionally, from July 1977, copies of all notifications for the register of the United Kingdom Children's Cancer 
Study Group (UKCCSG) have been sent to the Childhood Cancer Research Group. Confirmation of diagnosis and information on treatment and follow up were obtained from the hospitals at which the children were treated, from their family doctors or from the Medical Research Council Leukaemia Trials Office. Most of the deaths among these children are ascertained either through these records or through death certificates which are routinely sent to the Childhood Cancer Research Group for all children dying from malignant disease. Children not already known to have died have been flagged at the National Health Service Central Registers so that any deaths would automatically be notified to us, and have thus been followed up to the end of December 1986.

From all three sources of ascertainment combined, there were 4209 children with acute lymphoblastic leukaemia or acute unspecified or undifferentiated leukaemia who were domiciled in Great Britain at the time of diagnosis. Of these children 139 died before treatment could be started. The present analyses are based on the remaining 4070 children. For each child, the treatment centre was defined as the hospital from which the patient's chemotherapy was directed. Where a hospital closed during the period under review and treatment of childhood leukaemia was transferred to another hospital, or where treatment was administered by a team of clinicians working jointly at two hospitals, the treatment centre was taken to include both hospitals. The treatment centres were classified as follows according to the average number of patients in the series who were treated at them each year: A, average of at least six treated per year; B, average of at least one but fewer than six treated per year; and $\mathrm{C}$, average of fewer than one treated per year.

The results have been analysed using standard methods to examine the effects on survival of inclusion in the trials and of type of treatment centre. ${ }^{9}$

\section{Results}

Table 1 shows the numbers of children who were included in the Medical Research Council UKALL trials during the three year periods 1971-3, 1974-6, 1977-9, and 1980-2. The proportion of children included in the trials increased from $46 \%$ in $1971-3$ to $64 \%$ in $1977-9$. The proportion declined to $44 \%$ in 1980-2; this was largely because there was a gap of five months between the closure of UKALL VI and VII and the start of UKALL VIII, and because children at one very large centre were entered in a locally organised trial of maintenance treatment ${ }^{10}$ or
Table 1 The numbers of children treated inside and outside the UKALL trials and at centres of various sizes during 1971-82

\begin{tabular}{llllll}
\hline \multicolumn{3}{l}{ Year of first treatment } & Total \\
\cline { 2 - 4 } & $1971-82$
\end{tabular}

\begin{tabular}{lrrrrr}
\hline $\begin{array}{l}\text { In UKALL trials } \\
\text { Centre type: }\end{array}$ 萡 & & & & & \\
A & 422 & 331 & 374 & 281 & 1408 \\
B & 59 & 208 & 199 & 107 & 573 \\
C & 16 & 55 & 59 & 26 & 156 \\
Total & 497 & 594 & 632 & 414 & 2137 \\
& & & & & \\
Not in UKALL trials & & & & \\
Centre type: & & & & & \\
A & 144 & 269 & 215 & 327 & 955 \\
B & 246 & 124 & 97 & 142 & 609 \\
C & 201 & 77 & 43 & 48 & 369 \\
Total & 591 & 470 & 355 & 517 & 1933 \\
& & & & & \\
All patients & & & & & \\
Centre type: ${ }^{*}$ & & & & & \\
A & 566 & 600 & 589 & 608 & 2363 \\
B & 305 & 332 & 296 & 249 & 1182 \\
C & 217 & 132 & 102 & 74 & 525 \\
Total & 1088 & 1064 & 987 & 931 & 4070 \\
\hline
\end{tabular}

${ }^{*}$ Definition of type of centre: A, average of at least six patients treated per year; B, average of at least one and fewer than six patients treated per year; and $C$, average of fewer than one patient treated per year.

treated according to a pilot protocol for UKALL X. During 1977-82, some children with $T$ cell acute lymphoblastic leukaemia at certain centres were included in a UKCCSG trial of treatment for T cell lymphoma and leukaemia. ${ }^{11}$ The numbers of children treated at different categories of centre as defined in the methods section are also shown in table 1. The proportion treated at centres with an average of at least six patients per year increased from $52 \%$ of those diagnosed in $1971-3$ to $65 \%$ in 1980-2 while the proportion treated at centres with fewer than one patient per year declined from $20 \%$ to $8 \%$.

The figure shows the overall difference in survival for patients included and not included in the trials. The proportions of the two sexes and values of the white cell count, factors known to be of prognostic importance, were similar for the two groups of children. In the $0-1$ year age group, which has a poor prognosis, there was a smaller proportion of trial patients. When age was allowed for in the analysis, there was still a highly significant difference in survival rates in favour of the trial group $\left(\chi^{2}=102.5\right.$ on $1 \mathrm{df}$, stratified by period of diagnosis).

The survival rate was best for centres treating the 


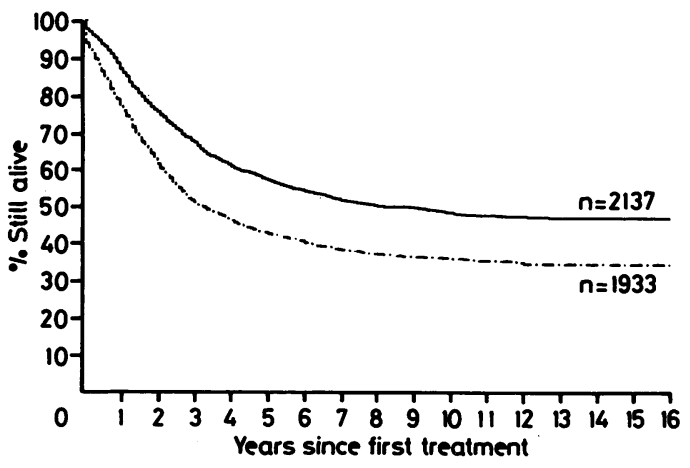

Figure Actuarial survival curves for children with acute lymphoblastic leukaemia treated 1971-82: children included (-) and not included (- - - ) in Medical Research Council trials. Log rank test for difference in survival rates, stratified by period of diagnosis and allowing for age: $p<0.0001$.

largest number of patients. The treatment centre groups were similar with respect to the sex ratio and the proportions of patients in different ranges of white cell counts. Children aged 10-14, who have a worse prognosis, were less likely to have been treated at a group A centre and correspondingly more likely to be in group $\mathrm{C}$, but when age was allowed for there was still a highly significant difference in survival rates in favour of larger centres $\left(\chi^{2}\right.$ for trend $=38.4$ on $1 \mathrm{df}$, stratified by period).

Effects of inclusion in the trials and of treatment at different types of centre were next considered together: for children included in the trials there was no difference in survival rates between centres of different size; for those not included in the trials, however, there was a noticeable pattern of higher survival rates at larger centres (table 2).

Changes over time in the five year survival rates for children first treated during 1971-82 are examined in table 2, in relation to inclusion in the trials and to size of centre. Overall there was an appreciable trend in rates from $37 \%$ for $1971-3$ to $66 \%$ for 1980-2. For children in the trials there was little change in survival rate during 1971-9, but for children not in the trials there was a substantial improvement. The rates for $1980-2$ showed a considerable improvement on 1971-9 for both trial and non-trial patients. Nevertheless the non-trial survival rate for 1980-2 had only reached that achieved for children included in the trials during the previous three years although, as already remarked, a substantial proportion of children not in the trials were treated at major centres. The difference in survival
Table 2 Five year actuarial survival rates (\%) for children treated inside and outside the UKALL trials and at centres of various sizes during 1971-82

\begin{tabular}{lllll}
\hline & \multicolumn{3}{l}{ Year of first treatment } \\
\cline { 2 - 5 } & $1971-3$ & $1974-6$ & $1977-9$ & $1980-2$ \\
\hline In UKALL trials & & & & \\
Centre type: $^{*}$ & & & & \\
A & 51 & 52 & 58 & 75 \\
B & 53 & 50 & 60 & 71 \\
C & 44 & 53 & 61 & 73 \\
All centres & 51 & 51 & 59 & 74 \\
& & & & \\
Not in UKALL trials & & & & \\
Centre type: & & & 50 & 60 \\
A & 30 & 49 & 44 & 63 \\
B & 28 & 40 & 33 & 50 \\
C & 20 & 38 & 46 & 60 \\
All centres & 26 & 45 & & \\
All patients & & & & \\
Centre type:* & & & & \\
A & 46 & 50 & 55 & 67 \\
B & 33 & 46 & 55 & 66 \\
C & 22 & 44 & 49 & 58 \\
All centres & 37 & 48 & 55 & 66 \\
\hline
\end{tabular}

${ }^{*}$ Definition of type of centre: A, average of at least six patients treated per year; B, average of at least one and fewer than six patients treated per year; and $C$, average of fewer than one patient treated per year.

between treatment centre groups for children not in the trials was observed throughout 1971-82. The improvement in survival for 1980-2 was observed for all types of centre.

The effects of inclusion in the trials and size of treatment centre were very noticeable during the few months immediately after diagnosis, and it might be argued that this reflects some selection bias; however, when patients who had died within three months of diagnosis were excluded from the analysis, the differences between trial and non-trial patients and between those at different categories of treatment centre were still highly significant.

Although the great majority of deaths from acute lymphoblastic leukaemia in children occur within five years of diagnosis, there is a substantial risk of death beyond this time. So far 248 deaths have been recorded among the 1835 children surviving at least five years up to the end of December 1986. The subsequent survival rate for those who survived five years did not differ as between trial and non-trial patients. This rate was, however, significantly higher for centres in group A than for groups B and C $\left(\chi^{2}=6.34\right.$ on $1 \mathrm{df}$, stratified by age and calendar period of diagnosis). 
Of the 248 patients who died after five years or more $189(76 \%)$ had suffered a first relapse at less than five years from diagnosis; $26(10 \%)$ had a first relapse later than five years (maximum nine years); seven $(3 \%)$ were in first remission from acute lymphoblastic leukaemia at the time of death; no information on time to first relapse was available for the remaining $26(10 \%)$.

As yet only 16 second primary tumours have been observed in the series; 13 of these 16 patients have died. There was no obvious association of the risk of developing a second primary tumour with whether children were included in the trials or with treatment centre size, but this result should be treated with caution because of the small number of second primary tumours as a proportion of the total series.

\section{Discussion}

There is considerable interest in questions concerning the centralisation of treatment for childhood cancer and leukaemia. Over the past 20 years there has been a continuing trend towards such centralisation in the United Kingdom; some indication of this is given in table 2 . There has been very little published evidence on the effects on the survival rate for childhood acute lymphoblastic leukaemia of such centralisation-that is, of treatment at specialist centres or within controlled clinical trials. In the mid 1960 s, when survival was generally extremely poor, it was found that children treated at specialist centres were more likely to survive. ${ }^{12}$ More recently, an analysis of over 300 children with acute lymphoblastic leukaemia from the population based Greater Delaware Valley Pediatric Tumor Registry in the United States showed significant advantages in survival for patients treated at specialist cancer centres and for those who were treated according to standardised protocols. ${ }^{13}$ The results of the present analyses agree with these findings. In the early 1970 s there were large differences in survival rates between centres treating many patients and those where only a few were treated. These differences diminished in more recent years. To some extent this may be explained by assuming that some of the less successful smaller centres gave up treating children with acute lymphoblastic leukaemia during this period. Also, of course, even the smallest centres which did continue to treat some of these children will have had a larger total number of patients by the 1980s.

In addition to the overall pattern of higher survival rates at treatment centres seeing larger numbers of patients, there was considerable variation in survival between individual major centres. Among the 12 centres in group $\mathrm{A}$, there was no association between survival rates and size of centre, but the five year survival rates of these centres ranged from $45 \%$ to $70 \%$. Some of this variation is probably explained by random differences in the proportions of children with good or poor prognostic features at individual centres. Nevertheless, detailed analysis of the rates achieved at one centre suggests that some of the variation may be due to differences in the application of apparently similar treatment protocols. ${ }^{14}$

Overall the survival rate at the group B centres was lower than for group $A$ and higher than for group C, but within group B there was no difference in rates between those centres with one, two, three, or four patients per year.

The effects on the survival rate of inclusion in the trials and size of treatment centre both persisted beyond three months from diagnosis. This suggests that the differences in survival rates cannot be explained solely by patients more frequently failing to achieve remission outside the trials and at minor centres.

The risk of first relapse after the completion of treatment generally appears to be low, ${ }^{12} 1516$ although first relapses have been recorded as long as nine years after entering remission both in the present series and previously. ${ }^{17}$ The Childhood Cancer Research Group registry does not hold details of the relapse free survival time of all children as this would require repeated active follow up of very large numbers of patients. The results presented here show no evidence that mortality rates among those surviving at least five years are different for trial and non-trial patients, though because of the appreciable differences during the first few years after diagnosis, those included in the trials had a substantially higher chance of becoming very long term survivors. The higher probability of survival for children treated at major centres appeared to persist even beyond the first five years after diagnosis.

We are grateful to our colleagues at the Childhood Cancer Research Group for help in various ways-in particular to $\mathrm{Mr}$ Harry Fearnley and Mrs E M Roberts for their work on the National Registry of Childhood Tumours on which this paper is largely based, and to Dr Michael Potok for help with computing. We thank the Medical Research Council Leukaemia Committee for providing information concerning children included in the trials, and individual members for useful discussions. We thank also the many consultants and general practitioners who provided the information on which this paper is based. We are grateful to the Office of Population Censuses and Surveys, the Information Services Division of the Common Services Agency of the Scottish Health Service, the General Register Office for Scotland, regional cancer registries and the UKCCSG for providing copies of notifications of childhood cancer cases. We thank the National Health Service Central Registers for notification of deaths and the flagging of survivors. Dr L M Kinnier Wilson kindly provided data from the Oxford Survey of Childhood Cancers. 
The Childhood Cancer Research Group is supported by the Department of Health and Social Security, the Scottish Home and Health Department, the Cancer Research Campaign, and the Leukaemia Research Fund. Collection of data was also supported by the Marie Curie Memorial Foundation.

\section{References}

${ }^{1}$ MRC Report to the Council by the Working Party on Leukaemia in Childhood. Analysis of treatment in childhood leukaemia. I. Predisposition to methotrexate-induced neutropenia after craniospinal irradiation. $\mathrm{Br}$ Med J 1975; iii:563-6.

2 MRC Report to the Council by the Working Party on Leukaemia in Childhood. Treatment of acute lymphoblastic leukaemia: effect of variation in length of treatment on duration of remission. Br Med J 1977;ii:495-7.

${ }^{3}$ MRC Report to the Council by the Working Party on Leukaemia in Childhood. Effects of varying radiation schedule, cyclophosphamide treatment, and duration of treatment in acute lymphoblastic leukaemia. Br Med J 1978;ii:787-91.

4 MRC Report of the Working Party on Leukaemia in Childhood. The treatment of acute lymphoblastic leukaemia (ALL) in childhood, UKALL III. Med Pediatr Oncol 1982;10:501-10.

5 MRC Report of the Working Party on Leukaemia in Childhood. Duration of chemotherapy in childhood acute lymphoblastic leukaemia. Med Pediatr Oncol 1982;10:511-20.

${ }^{6}$ MRC Report to the Council by the Working Party on Leukaemia in Childhood. UKALL V: an attempt to reduce immunosuppressive effects of therapy in childhood acute lymphoblastic leukemia. J Clin Oncol 1986;4:1758-64.

${ }^{7}$ MRC Report to the Council by the Working Party on Leukaemia in Childhood. Medical Research Council leukaemia trial UKALL VII. Arch Dis Child 1985;60:1050-4.

${ }^{8}$ MRC Report to the Council by the Working Party on Leukaemia in Childhood. Improvement in treatment for children with acute lymphoblastic leukaemia. Lancet 1986;i:408-11.

9 Peto R, Pike MC, Armitage P, et al. Design and analysis of randomised trials requiring prolonged observation of each patient. II. Analysis and examples. Br J Cancer 1977;35:1-39.

10 Chessells JM, Leiper AD, Tiedemann K, Hardisty RM, Richards S. Oral methotrexate is as effective as intramuscular in maintenance therapy of acute lymphoblastic leukaemia. Arch Dis Child 1987;62:172-6.

11 Mott MG, Chessells JM, Willoughby ML, et al. Adjuvant low dose radiation in childhood $\mathrm{T}$ cell lymphoma/leukaemia. $\mathrm{Br} \mathrm{J}$ Cancer 1984;50:457-62.

12 MRC Report to the Council from the Committee on Leukaemia and the Working Party on Leukaemia in Childhood. Duration of survival in children with acute leukaemia. $\mathrm{Br}$ Med J 1971;iv:7-9.

13 Meadows AM, Kramer S, Hopson R, Lustbader E, Jarrett P, Evans AE. Survival in childhood acute lymphocytic leukaemia: effect of protocol and place of treatment. Cancer Invest 1983;1:49-55.

14 Eden OB, Stiller CA, Gerrard MP. Improved survival for childhood acute lymphoblastic leukaemia: possible effect of protocol compliance. Pediatric Hematology and Oncology 1988; 5:83-91.

15 George SL, Aur RJA, Mauer AM, Simons JV. A reappraisal of the results of stopping therapy in childhood leukaemia. $N$ Engl $J$ Med 1979;300:269-73.

16 Chessells JM, Hardisty RM, Richards S. Long survival in childhood acute lymphocytic leukaemia. Br J Cancer 1987;55: 315-9.

17 Mandelli F, Amadori S, Ceci A, et al. Long-term survival in childhood acute lymphocytic leukaemia in Italy. Cancer 1981; 48:2364-7.

Correspondence and requests for reprints to $\mathrm{Mr} \mathrm{C} \mathrm{A}$ Stiller, Childhood Cancer Research Group, Radcliffe Infirmary, Oxford OX2 6HE.

Accepted 7 December 1987

\section{Addendum}

Since the above analyses were carried out, further cases of childhood acute lymphoblastic leukaemia have been ascertained by the Registry. Ascertainment is now virtually complete for $1980-4$, and most survivors have been followed up to the end of August 1988. There is thus now a greatly increased amount of data relating to children treated during the period of entry to UKALL VIII, a trial which used more sustained treatment than its predecessors and produced a higher survival rate. We have therefore analysed the data relating to trial entry, treatment centre size, and survival for children diagnosed in these years.

During 1980-4 there were 1558 children treated for acute lymphoblastic leukaemia, of whom $51 \%$ were included in the Medical Research Council trials. The great majority $(66 \%)$ were treated at group A centres, with $28 \%$ at group B, and only $5 \%$ at group $\mathrm{C}$. The overall five year survival rate was $68 \%$. Within the trials the five year survival rate was $72 \%$, compared with $64 \%$ outside the trials, and the difference between the survival curves was highly significant $\left(\chi^{2}=10.0\right.$ on $1 \mathrm{df}, \mathrm{p}<0.001$, allowing for age at diagnosis). For trial and non-trial patients combined, the five year survival rates at centres in groups A and B were very similar, $69 \%$ and $68 \%$ respectively, but the rate in group $C$ was only $53 \%$. There was no association between the survival rate and the number of patients per year within group B. Within the trials the survival rate was very similar at centres in groups A $(72 \%), \mathrm{B}(71 \%)$, and C (68\%). For non-trial patients, the rates were $64 \%, 66 \%$, and $48 \%$ respectively. These results show the continuing importance of treatment according to standardised protocols, particularly within nationally organised trials, for the achievement of the best survival rates in childhood acute lymphoblastic leukaemia. Since the 1970 s, the survival rates at centres treating between one and six new patients per year have become very similar to those obtained at the largest centres, but those for children treated at centres with fewer than one new patient per year and not included in the UKALL trials remained considerably poorer. 